\title{
Article \\ PAM50 Intrinsic Subtype Profiles in Primary and Metastatic Breast Cancer Show a Significant Shift toward More Aggressive Subtypes with Prognostic Implications
}

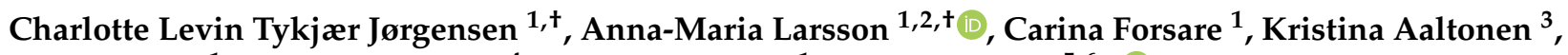 \\ Sara Jansson ${ }^{1}$, Rachel Bradshaw ${ }^{4}$, Pär-Ola Bendahl ${ }^{1}$ and Lisa Rydén ${ }^{5,6, *(D)}$
}

Citation: Jørgensen, C.L.T.; Larsson, A.-M.; Forsare, C.; Aaltonen, K.; Jansson, S.; Bradshaw, R.; Bendahl, P.-O.; Rydén, L. PAM50 Intrinsic Subtype Profiles in Primary and Metastatic Breast Cancer Show a Significant Shift toward More Aggressive Subtypes with Prognostic Implications. Cancers 2021, 13, 1592. https://doi.org/10.3390/ cancers13071592

Academic Editor: David Wong

Received: 19 February 2021

Accepted: 25 March 2021

Published: 30 March 2021

Publisher's Note: MDPI stays neutra with regard to jurisdictional claims in published maps and institutional affiliations.

Copyright: (c) 2021 by the authors. Licensee MDPI, Basel, Switzerland. This article is an open access article distributed under the terms and conditions of the Creative Commons Attribution (CC BY) license (https:// creativecommons.org/licenses/by/ $4.0 /)$.
1 Division of Oncology, Department of Clinical Sciences Lund, Lund University, SE-223 81 Lund, Sweden; charlotte.levin.tykjaer.joergensen@regionh.dk (C.L.T.J.); anna-maria.larsson@med.lu.se (A.-M.L.); carina.forsare@med.lu.se (C.F.); sara.jansson@med.lu.se (S.J.); par-ola.bendahl@med.lu.se (P.-O.B.)

2 Department of Hematology, Oncology and Radiation Physics, Skåne University Hospital, SE-221 85 Lund, Sweden

3 Division of Translational Cancer Research, Department of Laboratory Medicine, Lund University, SE-223 81 Lund, Sweden; kristina.aaltonen@med.lu.se

4 NanoString Technologies, Seattle, WA 98109, USA; rbradshaw@nanostring.com

5 Division of Surgery and Division of Oncology, Department of Clinical Sciences, Lund University, SE-223 81 Lund, Sweden

6 Department of Surgery, Skåne University Hospital, SE-205 02 Malmö, Sweden

* Correspondence: lisa.ryden@med.lu.se

$\dagger$ These authors contributed equally to this work.

Simple Summary: The majority of breast cancer deaths are caused by the spread of the disease to distant locations. The biological processes and molecular characteristics that eventually transform breast cancer into a life-threatening metastatic disease are not fully understood. The molecular subtyping of breast cancer into four tumor subtypes—namely luminal A, luminal B, human epidermal growth factor receptor 2-enriched, and basal-like subtypes-has been implemented for therapeutic guidance in patients with early breast cancer. It is not settled whether molecular subtypes in metastatic tissue can guide the choice of systemic therapy and how these subtypes may change throughout tumor progression. In this study, breast cancer subtypes at different stages of the disease were investigated, and we found changes to more unfavorable subtypes to be common throughout the progression of the disease. These findings suggests that molecular subtyping in metastatic disease could add important prognostic and predictive information to complement information from the primary tumor.

Abstract: Background: PAM50 breast cancer intrinsic subtyping adds prognostic information in early breast cancer; however, the role in metastatic disease is unclear. We aimed to identify PAM50 subtypes in primary tumors (PTs) and metastases to outline subtype changes and their prognostic role. Methods: RNA was isolated from PTs, lymph node metastases (LNMs), and distant metastases (DMs) in metastatic breast cancer patients $(n=140)$ included in a prospective study (NCT01322893). Gene expression analyses were performed using the Breast Cancer 360 (BC360) assay from NanoString. The subtype shifts were evaluated using McNemar and symmetry tests, and clinical outcomes were evaluated with log-rank tests and Cox regression. Results: The PAM50 subtype changed in 25/59 of paired samples between PTs and LNMs $\left(P_{\text {symmetry }}=0.002\right)$, in 31/61 between PTs and DMs $\left(P_{\text {symmetry }}<0.001\right)$, and in $16 / 38$ between LNMs and DMs $\left(P_{\text {symmetry }}=0.004\right)$. Shifts toward subtypes with worse outcomes were the most common. Patients with shifts from the luminal PT to non-luminal DM subtypes had worse progression-free survival compared to patients with a stable subtype (hazard ratio (HR): 2.3 ; 95\% confidence interval (CI): 1.14-4.68, $p=0.02$ ). Conclusion: Strong evidence of PAM50 subtype shifts toward unfavorable subtypes were seen between PTs and metastatic samples. For patients with a shift in subtype from luminal PT to non-luminal DM, a worse prognosis was noted. 
Keywords: metastatic breast cancer; PAM50 breast cancer intrinsic subtype; tumor progression; subtype shift; tumor heterogeneity

\section{Introduction}

The majority of breast cancer deaths can be attributed to metastasis [1]. Thus far, the biological processes and molecular characteristics of breast cancer's progression into life-threatening disease are still incompletely described. As personalized cancer treatment is based on tumor molecular characterization, tumor heterogeneity within the same patient causes great challenges in clinical practice [2-4]. A number of papers have reported a discrepancy in the status of routinely used biomarkers when applied to differentiate between the primary tumor and corresponding metastases in a relatively large proportion of metastatic breast cancer (MBC) patients [5-7]. The routine assessment of biomarker status using core needle biopsies from metastases are therefore recommended to improve the guidance for selecting systemic therapy in the context of metastasis [8].

Throughout tumor progression, little is known regarding changes in the four major molecular subtypes of breast cancer, namely the luminal A, luminal B, human epidermal growth factor receptor 2-enriched (HER2-E), and basal-like subtypes [9-17]. Based on relatively few studies, shifts in the breast cancer molecular subtypes in the range of $11-55 \%$ have been recognized when comparing patient-matched primary and metastatic tumors, with the majority of these changes being from the luminal A to non-luminal A subtypes. Differences between primary and recurrent disease could have an impact on the prognosis and, hence, have implications for the choice of systemic therapy. However, an unambiguous association has not been established between shifts in molecular traits and survival outcomes $[5,9,10,13,17]$. The previous assessments of change in tumor inherence were based on the analysis of archival tissues and mainly presented the surrogate subtypes $[5,9,10,17]$, although one of the studies presented data on the intrinsic molecular subtypes [13].

In the present study, we aimed to study changes in the intrinsic subtypes during tumor progression in breast cancer patients by analyzing the molecular status of tumor samples at different stages of the disease. The main objective was to analyze tumor specimens from a prospective observational trial of $\mathrm{MBC}$ patients with long-term follow-up data to determine the concordance of PAM50 breast cancer intrinsic subtypes in matched samples of primary tumors (PTs), synchronous lymph node metastases (LNMs), and distant metastases (DMs). A secondary objective was to evaluate the effect of subtype instability on prognosis and to assess the prognostic value of the subtype classification of metastases.

\section{Materials and Methods}

\subsection{Patients and Tissue Samples}

The design of the current study was prospective-retrospective [18] and used a patient cohort enrolled in a prospective monitoring trial (circulating tumor cell (CTC)-MBC trial; Clinical Trials.gov NCT01322893) to evaluate the prognostic value of serial enumeration of CTCs in women with newly diagnosed MBC scheduled for first-line systemic therapy [19]. In brief, the inclusion criteria were a diagnosis of $\mathrm{MBC}$, age $\geq 18$ years, an Eastern Cooperative Oncology Group (ECOG) performance status score of $0-2$, and a predicted life expectancy of $>2$ months.

Patients were excluded if they were unable to understand the study information, if they had received prior systemic treatment for metastatic disease, or if they had been diagnosed with any other malignant disease within the previous five years. Informed consent was obtained from all patients included in the study. The Lund University Ethics Committee (LU 2010/135) approved the study prior to its initiation. Of the 168 patients initially included in the trial, eight patients were excluded because they did not fulfill the inclusion criteria and four patients were excluded because they only had loco-regional 
recurrence. Based on pathology reports of the remaining 156 patients, formalin-fixed, paraffin-embedded (FFPE) samples of PTs, synchronous LNMs, and DMs were collected. In cases where multiple DMs were available, the sample from the first metastatic site was used in analysis (Figure 1).
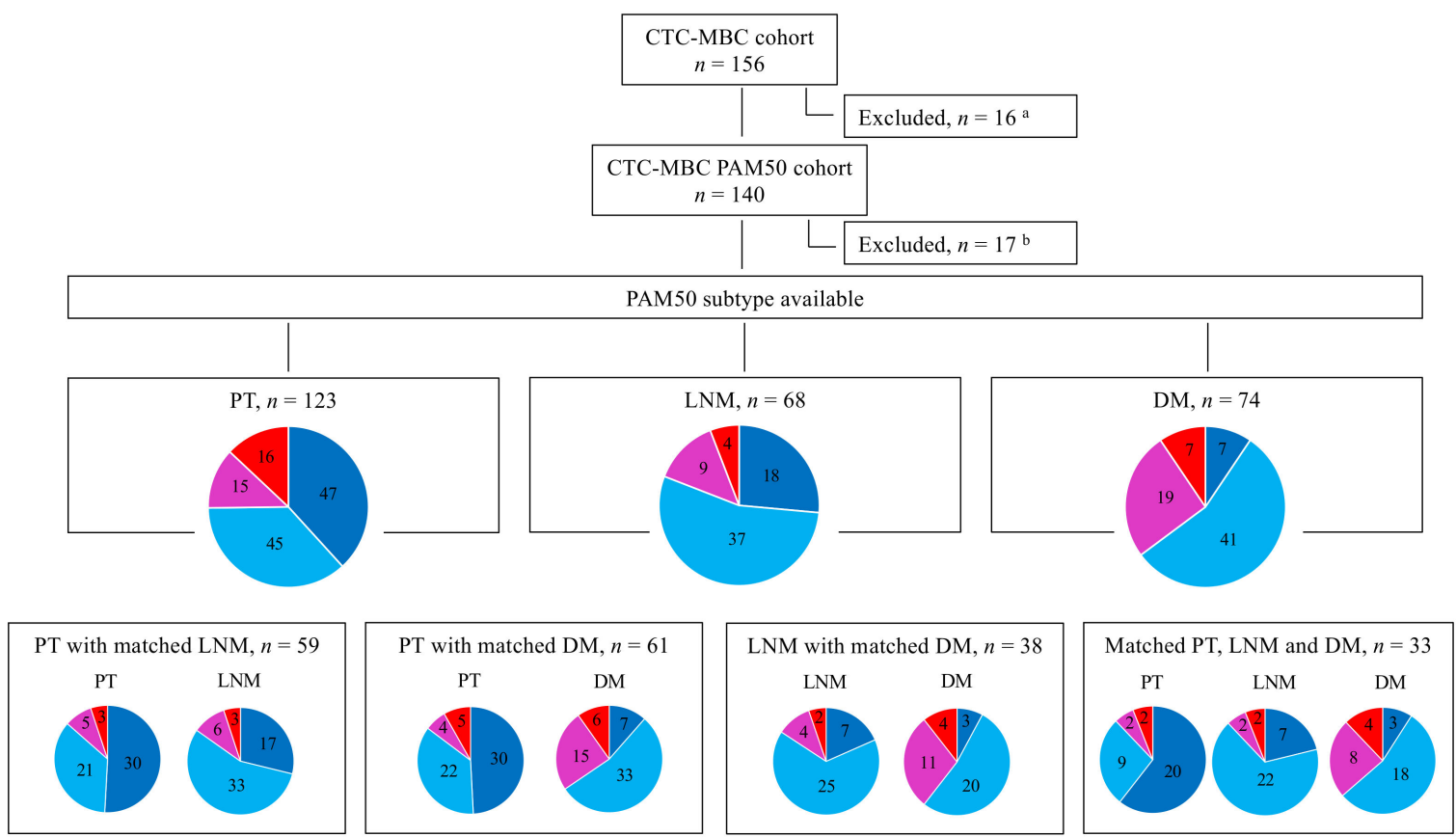

- Luminal A - Luminal B |- HER2-enriched a Basal

Figure 1. Flow chart of the study population and PAM50 breast cancer intrinsic subtype distribution between tumor sites. ${ }^{a}$ Patients were excluded from the study population due to any of the following reasons: sample being unavailable, no tumor tissue in the available sample, only locoregional sample available, or unsuccessful RNA extraction. ${ }^{b}$ Patients were excluded due to unsuccessful NanoString Breast Cancer 360 (BC360) assay or because PAM50 subtypes could not be assigned. Abbreviations: CTC: circulating tumor cell; DM: distant metastasis; HER2: human epidermal growth factor receptor 2; LNM: lymph node metastasis; MBC: metastatic breast cancer; PT: primary tumor.

\subsection{Macrodissection of Tumor Tissue and RNA Isolation}

Sections from collected FFPE tumor tissues stained with hematoxylin and eosin were reviewed, and areas with representative invasive breast carcinoma were outlined on each slide. In doubtful cases, a pathologist (Anna Ehinger) performed a second assessment. Based on the size of the tumor surface area, a total of 1-8 tissue sections, $10 \mu \mathrm{m}$ thick, were cut and macrodissected to remove the surrounding normal tissue outside the outlined area.

Total RNA was extracted using the AllPrep DNA/RNA FFPE kit (Qiagen Cat: 80234, Hilden, Germany) in accordance with the instructions provided by the manufacturer. RNA yield and purity were assessed using a NanoDrop ND-8000 Spectrophotometer (NanoDrop Technologies, Rockland, DE, USA). RNA quality control (QC) was performed using an Agilent 2100 Bioanalyzer Instrument (Agilent Technologies, Santa Clara, CA, USA). A subset of samples was analyzed with the Bioanalyzer as part of the QC of the RNA (mean RNA integrity number (RIN)-value was 2.3 (1.3-2.8). NanoString used multiple QC cut-offs to determine if a sample was PASS, FAIL, or BORDERLINE, which indicated if the RNA quality was high enough to produce reliable subtypes and that the NanoString assay should perform as expected. Due to these post-collection QC steps, NanoString did not need to assess RNA quality before the assay. Most samples (90\%) passed the predefined quality criteria set by NanoString, and only 17 patients were excluded due to analytical failure (Figure 1). 


\subsection{Gene Expression Assay}

Gene expression was measured using the NanoString Breast Cancer 360 assay $\left(\mathrm{BC} 360^{\mathrm{TM}}\right)$ on an NanoString nCounter ${ }^{\circledR}$ SPRINT Profiler (NanoString Technologies Inc., Seattle, WA, USA). The BC360 assay covers genes from 33 independent signatures, including the PAM50 signature (https:/ / www.nanostring.com/, accessed date: 30 August 2019). By using multiplexed hybridization and digital readouts of fluorescent barcoded probes, the NanoString platform measures the relative abundance of each mRNA transcript of interest [20]. In a single reaction, the $\mathrm{BC} 360^{\mathrm{TM}}$ gene expression panel (including 758 genespecific probe pairs of the $\mathrm{BC} 360$ targets, 18 housekeeping genes used for normalization, 6 exogenous positive control RNA targets, and 8 exogeneous negative control sequences) was hybridized in solution with $50-250 \mathrm{ng}$ total RNA overnight $(24 \mathrm{~h})$ at $65^{\circ} \mathrm{C}$. The samples were processed using the NanoString nCounter ${ }^{\circledR}$ SPRINT platform in accordance with the instructions and kits provided by NanoString Technologies (https:/ / www.nanostring. com/, accessed date: 30 August 2019).

\subsection{Molecular Subtype Classification}

Reporter code count files containing the counts of each target mRNA molecule for every sample were sent to NanoString Technologies Inc. (Seattle, WA, USA). The raw data underwent a quality control check to ensure that PAM50 subtypes could be calculated. Only sample data that passed predefined quality control thresholds for the housekeeping gene geomean and the housekeeping gene signal-to-noise ratio were included in the PAM50 analysis. PAM50 breast cancer intrinsic subtype calling was conducted using a proprietary algorithm based on the 50-gene expression signature detailed by Parker et al. [21], ultimately classifying the samples into the four following subtypes: luminal A, luminal B, HER2-E, and basal-like. For each sample, the Pearson's correlation coefficient for each of the four PAM50 centroids was calculated, and the subtype of the sample was assigned to the subtype of the centroid with the highest correlation.

\subsection{Statistical Analysis}

The associations between the PAM50 breast cancer intrinsic subtype and different patient and tumor characteristics were analyzed with Pearson's chi-square test or Fisher's exact test if the lowest expected count in a contingency table cell was $<5$. The exact McNemar test was used for comparisons of dichotomized PAM50 breast cancer intrinsic subtype status at two locations. When comparing all four subtypes at two locations, an exact test of symmetry was used. The null hypothesis of symmetry corresponded to a balanced subtype shift, and the alternative hypothesis corresponded to skewness in the shifts. Sankey diagrams were used to depict subtype shifts between the different locations.

The endpoints of the original trial were used to evaluate the prognostic value of the PAM50 breast cancer intrinsic subtype classification at each tumor site and PAM50 breast cancer intrinsic subtype shift versus no shift. The primary endpoint was the progressionfree survival (PFS), and the secondary endpoint was the overall survival (OS) [19]. The progression-free survival was calculated from when the blood sample was collected on the day of inclusion in the study until the day that progression was diagnosed by radiology and/or clinical assessment. The follow-up time was censored at the date of the last followup for patients who did not reach the outcome event.

To evaluate the association between subtype shift and prognosis, Kaplan-Meier estimates and log-rank tests were used. Hazard ratios (HRs) were calculated using Cox regression. Multivariable Cox models were used to evaluate whether the PAM50-related variables added prognostic value to the prognostic variables suggested by Bidard et al. [22] The variables included in the multivariable models were the following: age at diagnosis of metastatic breast cancer ( $<65$ versus $\geq 65$ years), ECOG performance status ( 0 versus 1 versus 2), Nottingham histologic grade (NHG) of PT (I versus II versus III), metastasis-free interval (MFI) ( 0 versus $>0-3$ versus $>3$ years), number of metastatic sites $(<3$ versus $\geq 3$ ), 
site of metastasis (visceral versus non-visceral), and CTC status ( $<5$ versus $\geq 5$ CTC). The proportional hazards assumptions for the Cox models were checked graphically.

All presented statistical tests were two-sided. The $p$-values, which should be interpreted as the level of evidence against the null hypothesis, were not adjusted for multiple testing. Following the work of Benjamin et al. [23], the term 'suggestive evidence' was used for $p$-values in the range of $0.005-0.05$, and the term 'significant evidence' was used for $p$-values below 0.005. Statistical calculations were conducted using IBM SPSS Statistics (version 24.0 and 26.0, IBM, Armonk, NY, USA) and STATA (version 16.1, StataCorp., College Station, TX, USA). The results are presented in accordance with the Reporting Recommendations for Tumor Marker Prognostic Studies (REMARK) where applicable [24,25].

\section{Results}

\subsection{Patients and Tumor Characteristics}

The original study included patients with newly diagnosed MBC who were previously untreated for metastatic disease. Of the 156 patients included in the original observational study, 140 (90\%) were included in the final PAM50 analysis (Figure 1). The patient demographics, disease characteristics, and prior therapy for the original cohort and the included versus excluded subgroups are presented in Table S1.

Overall, the characteristics of the 140 patients included in the present study were representative of the original cohort. However, non-assessable patients did tend to present with smaller PTs, less lymph node involvement, and fewer metastatic sites. The median follow-up time from inclusion in the study to progression was 49 months (range of 27-93 months) for patients alive at their last medical visit before database lock (April 2019). At this time point, $7 \%$ of the patients were progression-free and $29 \%$ were still alive. Twentynine patients presented with de novo MBC at the time of inclusion in the study, whereas 111 patients were diagnosed with distant recurrence.

\subsection{PAM50 Breast Cancer Intrinsic Subtype Distribution and Association with Clinicopathologic Characteristics}

The PAM50 breast cancer intrinsic subtypes could be assigned to 123 PTs, 68 LNMs, and $74 \mathrm{DMs}$, of which 33 patients had matched samples from all three locations (Figure 1 and Table 1). The characteristics of the PTs of the patients $(n=123)$ with the assignment of a PAM50 intrinsic molecular subtype were in line with those PTs included in the original study and the initial PAM50 cohort (Table S1). The available DM biopsies were obtained from the following sites: bone $(n=43)$, liver $(n=17)$, skin $(n=3)$, central nervous system (CNS; $n=2)$, lung $(n=2)$, pleura $(n=2)$, stomach $(n=2)$, ovary $(n=1)$, bladder $(n=1)$, and cervix $(n=1)$. The distribution of the PAM50 breast cancer intrinsic subtypes determined for PTs and metastases (LNMs and DMs) is shown in Figure 1.

Overall, the most frequent PT subtypes were luminal A $(38 \%, 47 / 123)$ and luminal B $(37 \%, 45 / 123)$, followed by basal-like $(13 \%, 16 / 123)$ and HER2-E $(12 \%, 15 / 123)$. Regarding the subtype assignment for LNMs, luminal B was the most common subtype $(54 \%, 37 / 68)$, followed by luminal A $(26 \%, 18 / 68)$. Most DMs were also classified as luminal B ( $55 \%$, $41 / 74)$, while the second most common DM subtype was HER2-E $(26 \%, 19 / 74)$. Only $9 \%$ $(7 / 74)$ of DMs were classified as luminal A.

Patient and tumor characteristics according to the PAM50 intrinsic subtypes in PTs, LNMs, and DMs are summarized in Table 1 and Supplementary Tables S2 and S3, respectively. Evidence for an association with the PT PAM50 subtype was seen for the nodal status $(p=0.01)$, MFI $(p=0.02)$, NHG, estrogen receptor $(\mathrm{ER})$, progesterone receptor $(\mathrm{PR})$, and HER2-status, as well as for the clinically defined immunohistochemistry (IHC) molecular subtype (all $p$-values $<0.001$ ).

Patients with the luminal A PT subtype were more frequently observed to have a longer MFI ( $76 \%,>3$ years) compared to the other subtypes. Conversely, patients with a basal-like subtype PT generally had a shorter MFI ( $44 \% \geq 0-3$ years) (Table 1$)$. Patients with 
de novo metastatic disease were found in all PAM50 intrinsic subtypes but most commonly presented with the luminal B subtype $(42 \%, 11 / 26$; Table 1$)$.

Table 1. Patient and tumor characteristics for primary tumors in the PAM50 cohort and subdivided into patients with subtype available and the different breast cancer intrinsic subtypes.

\begin{tabular}{|c|c|c|c|c|c|c|}
\hline Characteristics & $\begin{array}{l}\text { PAM50 Subtype } \\
\text { Available } n(\%)\end{array}$ & Lum A $n(\%)$ & Lum B $n(\%)$ & HER2-E $n(\%)$ & Basal-Like $n(\%)$ & $p^{a}$ \\
\hline All & 123 & 47 & 45 & 15 & 16 & \\
\hline $\begin{array}{c}\text { Age at MBC diagnosis } \\
\text { (years) }\end{array}$ & & & & & & $0.20^{\mathrm{d}}$ \\
\hline$<65$ & $63(51)$ & $22(47)$ & $20(44)$ & $10(67)$ & $11(69)$ & \\
\hline$\geq 65$ & $60(49)$ & $25(53)$ & $25(56)$ & $5(33)$ & $5(31)$ & \\
\hline ECOG (BL) & & & & & & $0.10^{\mathrm{e}}$ \\
\hline 0 & $70(58)$ & $34(75)$ & $21(48)$ & $7(47)$ & $8(50)$ & \\
\hline 1 & $31(26)$ & $8(18)$ & $14(32)$ & $5(33)$ & $4(25)$ & \\
\hline 2 & $19(16)$ & $3(7)$ & $9(20)$ & $3(20)$ & $4(25)$ & \\
\hline Missing & 3 & 2 & 1 & 0 & 0 & \\
\hline Size (PT) & & & & & & $0.77^{\mathrm{e}}$ \\
\hline $\mathrm{T} 1$ & $38(33)$ & $15(33)$ & $13(29)$ & $3(27)$ & $7(43)$ & \\
\hline $\mathrm{T} 2$ & $43(37)$ & $17(38)$ & $18(40)$ & $5(46)$ & $3(19)$ & \\
\hline T3 & $19(16)$ & $9(20)$ & $6(13)$ & $1(9)$ & $3(19)$ & \\
\hline $\mathrm{T} 4$ & $17(14)$ & $4(9)$ & $8(18)$ & $2(18)$ & $3(19)$ & \\
\hline Missing & 6 & 2 & 0 & 4 & 0 & \\
\hline Nodal status ${ }^{b}$ & & & & & & $0.01^{\mathrm{e}}$ \\
\hline Negative & $31(29)$ & $7(17)$ & $11(27)$ & $4(36)$ & $9(64)$ & \\
\hline Positive & $77(71)$ & $35(83)$ & $30(73)$ & $7(64)$ & $5(36)$ & \\
\hline Missing & 15 & 5 & 4 & 4 & 2 & \\
\hline NHG (PT) & & & & & & $<0.001{ }^{\mathrm{e}}$ \\
\hline I & $8(8)$ & $7(18)$ & $1(3)$ & 0 & 0 & \\
\hline II & $50(51)$ & $28(72)$ & $15(39)$ & $6(60)$ & $1(9)$ & \\
\hline III & $40(41)$ & $4(10)$ & $22(58)$ & $4(40)$ & $10(91)$ & \\
\hline Missing & 25 & 8 & 7 & 5 & 5 & \\
\hline ER (PT) & & & & & & $<0.001^{\mathrm{e}}$ \\
\hline Negative & $20(17)$ & $1(2)$ & $1(2)$ & $6(40)$ & $12(75)$ & \\
\hline Positive & $99(83)$ & $43(98)$ & $43(98)$ & $9(60)$ & $4(25)$ & \\
\hline Missing & 4 & 3 & 1 & 0 & 0 & \\
\hline PR (PT) & & & & & & $<0.001^{\mathrm{e}}$ \\
\hline Negative & $37(32)$ & $4(10)$ & $9(21)$ & $10(67)$ & $14(88)$ & \\
\hline Positive & $80(68)$ & $38(90)$ & $35(79)$ & $5(33)$ & $2(12)$ & \\
\hline Missing & 6 & 5 & 1 & 0 & 0 & \\
\hline HER2 (PT) & & & & & & $<0.001{ }^{\mathrm{e}}$ \\
\hline Negative & $89(86)$ & $32(94)$ & $36(88)$ & $7(50)$ & $14(100)$ & \\
\hline Positive & $14(14)$ & $2(6)$ & $5(12)$ & $7(50)$ & 0 & \\
\hline Missing & 20 & 13 & 4 & 1 & 2 & \\
\hline
\end{tabular}


Table 1. Cont.

\begin{tabular}{|c|c|c|c|c|c|c|}
\hline Characteristics & $\begin{array}{l}\text { PAM50 Subtype } \\
\text { Available } n(\%)\end{array}$ & Lum A $n(\%)$ & Lum B $n(\%)$ & HER2-E $n(\%)$ & Basal-Like $n(\%)$ & $p^{\mathrm{a}}$ \\
\hline BC subtype (PT) & & & & & & $<0.001^{\mathrm{e}}$ \\
\hline ER+ HER2- & $75(73)$ & $32(94)$ & $35(88)$ & $4(29)$ & $4(29)$ & \\
\hline HER2+ (ER+/ER-) & $13(13)$ & $2(6)$ & $4(10)$ & $7(50)$ & 0 & \\
\hline ER - HER2- & $14(14)$ & 0 & $1(2)$ & $3(21)$ & $10(71)$ & \\
\hline Missing & 21 & 13 & 5 & 1 & 2 & \\
\hline MFI (years) & & & & & & $0.02^{\mathrm{e}}$ \\
\hline $0^{c}$ & $26(21)$ & $7(15)$ & $11(24)$ & $5(33)$ & $3(19)$ & \\
\hline$>0-3$ & $23(19)$ & $4(9)$ & $8(18)$ & $4(27)$ & $7(44)$ & \\
\hline$>3$ & $74(60)$ & $36(76)$ & $26(58)$ & $6(40)$ & $6(37)$ & \\
\hline $\begin{array}{c}\text { Number of metastatic } \\
\text { sites }\end{array}$ & & & & & & $0.70^{\mathrm{d}}$ \\
\hline $1-2$ & $82(67)$ & $33(70)$ & $30(67)$ & $8(53)$ & $11(69)$ & \\
\hline$\geq 3$ & $41(33)$ & $14(30)$ & $15(33)$ & $7(47)$ & $5(31)$ & \\
\hline Metastatic site & & & & & & $0.26^{\mathrm{d}}$ \\
\hline Non-visceral & $51(42)$ & $23(49)$ & $19(42)$ & $3(20)$ & $6(38)$ & \\
\hline Visceral & $72(58)$ & $24(51)$ & $26(58)$ & $12(80)$ & $10(62)$ & \\
\hline Number of CTCs (BL) & & & & & & $0.32^{d}$ \\
\hline$<5$ & $57(46)$ & $25(53)$ & $16(36)$ & $7(47)$ & $9(56)$ & \\
\hline$\geq 5$ & $66(54)$ & $22(47)$ & $29(64)$ & $8(53)$ & $7(44)$ & \\
\hline Missing & 0 & 0 & 0 & 0 & 0 & \\
\hline
\end{tabular}

Abbreviations: BL: baseline; BC: breast cancer; CTCs: circulating tumor cells; ECOG: Eastern Cooperative Oncology Group; ER: estrogen receptor; HER2: human epidermal growth factor receptor 2; HER2-E: human epidermal growth factor receptor 2 enriched; Lum A/B: luminal A/B; MBC: metastatic breast cancer; MFI: metastasis-free interval; NHG: Nottingham Histological Grade; $n$ : number; PT: primary tumor; PR: progesterone receptor. ${ }^{\mathrm{a}}$ Test of homogeneity across subtypes, missing value category excluded; ${ }^{\mathrm{b}}$ at primary diagnosis; ${ }^{\mathrm{c}}$ represents de novo MBC; ${ }^{\mathrm{d}} p$-value from Pearson's chi-square test; ${ }^{\mathrm{e}} p$-value from Fisher's exact test.

\subsection{Concordance between PAM50 Breast Cancer Intrinsic Subtypes and Clinically Defined ER and HER2 Expression Status}

Excellent agreement was found between the PT ER-positive IHC status and luminal subtypes (A and B) in PTs (98\% for both, 43/44; Table 1 and Table S2). When comparing the HER2-positive status in PTs and the HER2-E intrinsic subtype in PTs, half of the tumor samples categorized as HER2-E intrinsic subtypes had HER2-positive status in the clinical pathological analyses of PTs $(50 \%, 7 / 14)$, whereas four were ER-positive/HER2-negative, and three were triple-negative (TNBC). In line with these findings, HER2-positive tumors were categorized as being of the luminal B molecular subtype in 36\% (5/14) of PTs.

Comparing intrinsic subtypes and the clinically assessed biomarker status of DMs revealed an excellent agreement (7/7 versus $35 / 35$, i.e., 100\%) between DMs with ERpositive IHC status and the luminal A and B subtypes (Table S3). Comparing DMs and HER2-positive status with the intrinsic subtype showed that only $33 \%(6 / 18)$ of HER2-E DMs had a clinically defined HER2-positive tumor. Regarding all HER2-positive DMs, 75\% $(6 / 8)$ were categorized as being of the HER2-E subtype, whereas one was categorized as luminal $\mathrm{B}$ and one as basal-like.

\subsection{PAM50 Breast Cancer Intrinsic Subtype Status across Tumor Progression Stages in Matched Samples}

The PAM50 breast cancer intrinsic subtype distribution of matched samples is displayed in Figure 1, and changes in the matched tumor pairs and triplets are presented in Figure 2A-D and in Table S4. Paired data between PTs and LNMs were available for a total of 59 cases for the PAM50 intrinsic subtype (Figure 2A) and for a total of 61 cases between PTs and DMs (Figure 2B). 


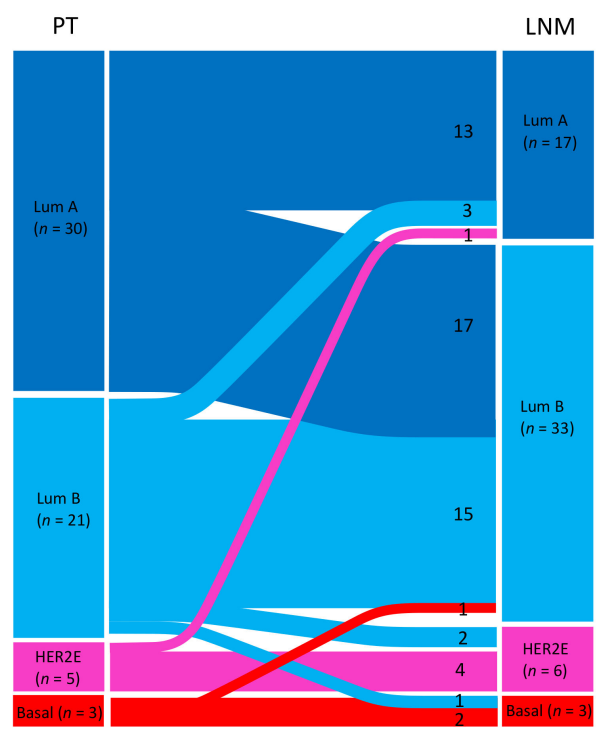

(A)

PT

DM

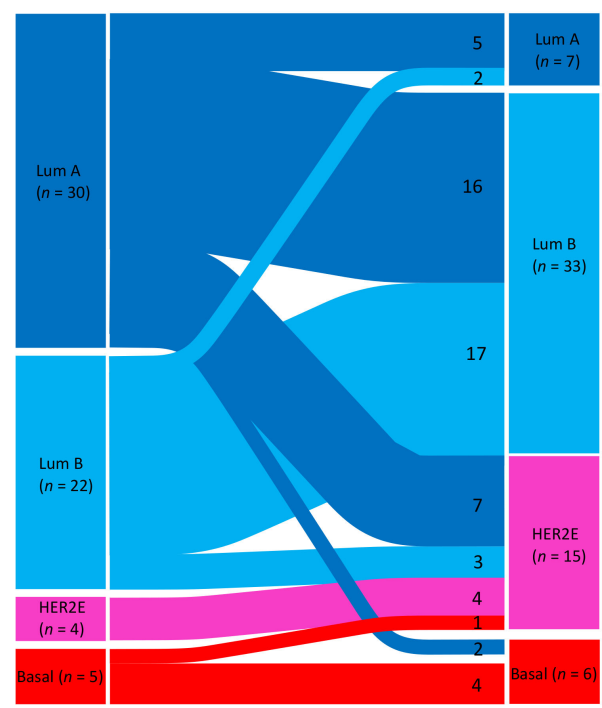

(B)

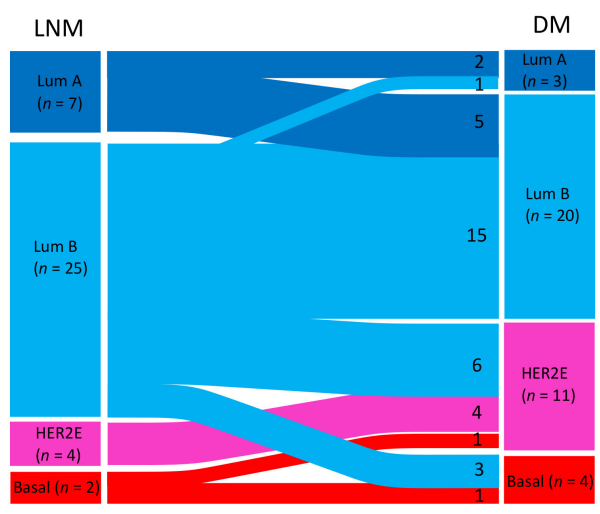

(C)

Figure 2. Cont. 


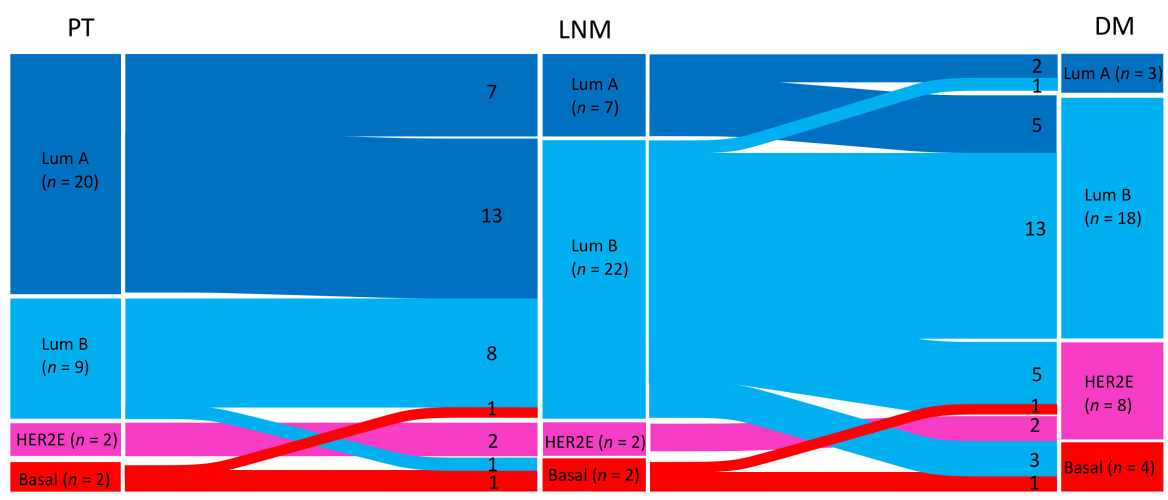

(D)

Figure 2. (A-D): Sankey diagrams showing the PAM50 breast cancer intrinsic subtype shifts of matched samples between (A) the primary tumor (PT) and lymph node metastasis (LNM) $(n=59)$; (B) the PT and distant metastasis (DM) $(n=61)$; (C) LNM and DM ( $n=38)$; and (D) the PT, LNM, and DM $(n=33)$. Abbreviations: HER2-E: human epidermal growth factor receptor 2-enriched; Lum A/B: luminal A/B.

In PTs with matching LNM samples $(n=59), 51 \%(30 / 59)$ were classified as luminal A, whereas only $29 \%$ (17/59) of matched LNMs were assigned the luminal A subtype. In paired samples from PTs and DMs $(n=61), 49 \%(30 / 61)$ of PTs were classified as luminal A, whereas only $11 \%(7 / 61)$ of matched DMs were luminal A. In samples with matched PTs, LNMs, and DMs $(n=33), 61 \%(20 / 33)$ of PTs were luminal A, but only $21 \%(7 / 33)$ of LNMs and $9 \%(3 / 33)$ of DMs were classified the same (Figure 1).

\subsubsection{Primary Tumors versus Matched Samples of Lymph Node Metastases}

In matched samples of PTs and LNMs $(n=59)$, PAM50 breast cancer intrinsic subtype shifts were seen in $42 \%$ (25/59) of cases across all molecular subtypes (Figure 2A, Table S4), and this finding was shown to be significantly skewed using the exact test of symmetry $(p=0.002)$. Shifts were seen in both directions; however, the most common shift was from the luminal A subtype to the luminal B subtype. In exact McNemar tests, a shift in subtype was seen in $36 \%$ (21/59) of cases considering luminal A versus not, where $81 \%(17 / 21)$ of case switched from the luminal A subtype to the luminal B subtype $(p=0.007)$.

\subsubsection{Primary Tumors versus Matched Samples of Distant Metastases}

In matched samples of PTs and DMs $(n=61)$, shifts in subtype were seen in $51 \%$ $(31 / 61)$ of cases (Figure 2B, Table S4), and the shifts were shown to be significantly skewed by the exact test of symmetry $(p<0.001)$. The most common shift $(n=25)$ was from the luminal A PT subtype, with 16 cases shifting to luminal B, seven cases shifting to HER2E, and two cases shifting to the basal DM (McNemar test, $p<0.001)$.

\subsubsection{Lymph Node Metastases versus Matched Samples of Distant Metastases}

Matched samples of LNMs and DMs were available for a total of 38 cases for the PAM50 subtype, and shifts in subtype were noted in $42 \%(16 / 38)$ of cases (Figure 2C, Table S4). These shifts were found to be significantly skewed $(p=0.004)$. Between LNMs and DMs, the luminal B subtype changed in $40 \%(10 / 25)$ of cases; of these, shifts to the HER2-E or basal-like subtype were seen in $90 \%(9 / 10)$ of cases (Table S4). For these 10 pairs with the luminal B subtype shift, the DMs were located at different sites (four in bone, two in the liver, one in the skin, one in the CNS, one in the ovaries, and one in the urinary bladder). Five of seven LNMs with the luminal A subtype switched to the luminal B subtype in DMs. 
3.4.4. Primary Tumors versus Matched Samples of Lymph Node Metastases and Distant Metastases

Thirty-three patients had matched samples from all three sites (PT, LNM, and DM). Overall, 64\% (21/33) of the patients showed a shift in their PAM50 subtype, and this was toward a more unfavorable subtype for most of them $(86 \%, 18 / 21)$ (Figure 2D and Table S4).

\subsection{Comparing PAM50 Breast Cancer Intrinsic Subtype Shifts from Luminal to Non-Luminal Subtypes between Tumor Sites}

When comparing shifts from luminal (luminal A or luminal B) to non-luminal (HER2E or basal-like) subtypes between tumor sites, differences were seen regarding shifts from PT to LNM versus shifts from PT to DM. A shift from the PT luminal subtype to the non-luminal subtype in LNMs was detected in only $6 \%(3 / 51)$ of patients (Figure $2 \mathrm{~A}$, Table S4) compared to 23\% (12/52) between PTs and DMs (Figure 2B, Table S4). Between PTs and DMs, $40 \%(12 / 30)$ of shifts involved a change from the luminal subtype to the non-luminal subtype (exact McNemar test $p<0.001$ ). Of these, 10/12 shifted to the HER2-E DM subtype (Figure 2B). A comparison between LNMs and DMs showed a shift from luminal to non-luminal in 24\% $(9 / 38$; exact McNemar test $p=0.004)$ of cases, in which six cases with the LNM luminal (B) subtypes switched to the HER2-E subtype in DMs and three shifted to the basal-like subtype.

\subsection{Associations between PAM50 Breast Cancer Intrinsic Subtype Shifts and Adjuvant Therapy}

For patients that received adjuvant chemotherapy versus those who did not, there was no significant difference between patients with a stable subtype and those with subtype shifts (Table S5). When considering subtype shifts from PT to DM in patients that received adjuvant endocrine therapy, there was a shift in $61 \%(27 / 44)$ of cases compared with $24 \%$ $(4 / 17)$ of patients who did not receive adjuvant endocrine therapy $(p=0.01$; Table S5).

\subsection{PAM50 Breast Cancer Intrinsic Subtype Status and Outcome in Patients with Available Subtype Assignment in PT, LNM, and DM}

The PAM50 breast cancer intrinsic subtype of the PTs $(n=123)$ was significantly associated with PFS $(p=0.001)$ and OS $(p<0.001)$ for metastatic disease (Figure 3A,B). Patients assigned the PT basal-like subtype had worse outcomes than those with the other subtypes (median PFS of 5 months versus 11-18 months, respectively, and median OS of 11 months versus $35-45$ months, respectively). When analyzing the LNM intrinsic subtype $(n=68)$ and the correlation with survival, there was suggestive evidence for an association with PFS ( $p=0.027)$ but not OS ( $p=0.058$; Figure 3C,D), and DM intrinsic subtypes $(n=74)$ had no significant associations with PFS or OS $(p=0.096$ and $p=0.907$, respectively; Figure 3E,F).

In Cox proportional hazards models, the PAM50 subtype of the PTs remained an independent prognostic factor for both PFS ( $p=0.007)$ and OS $(p=0.001)$ in multivariable models adjusted for clinically relevant prognostic factors (Table 2). Regarding the LNM intrinsic subtype, there were no significant correlations with PFS or OS in the adjusted Cox regression models. However, in the adjusted analyses, a significant difference in PFS was observed when the PAM50 intrinsic subtypes of the DMs were considered $(p=0.002$; 3-degree-of-freedom (df) overall test), and there was suggestive evidence for a correlation with OS (3-df $p=0.05$; Table 2). 
Primary Tumor

A

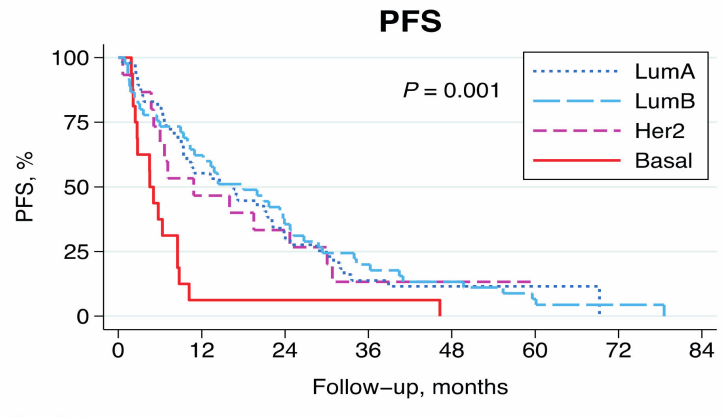

B

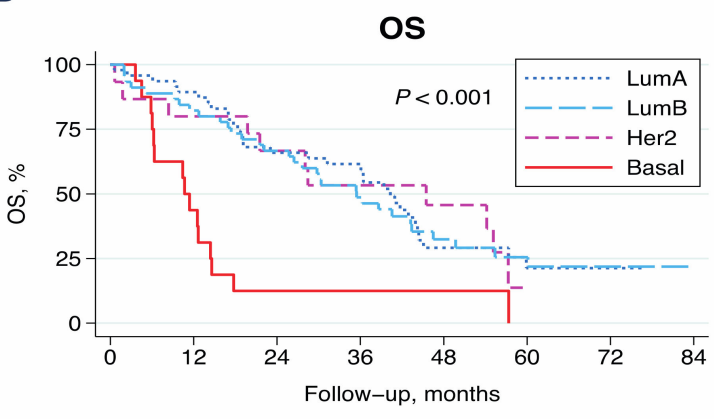

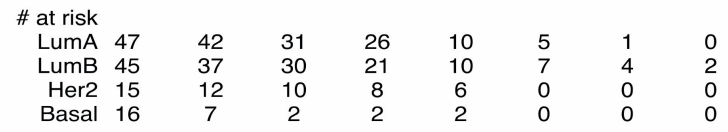

\section{Lymph Node Metastasis}

C

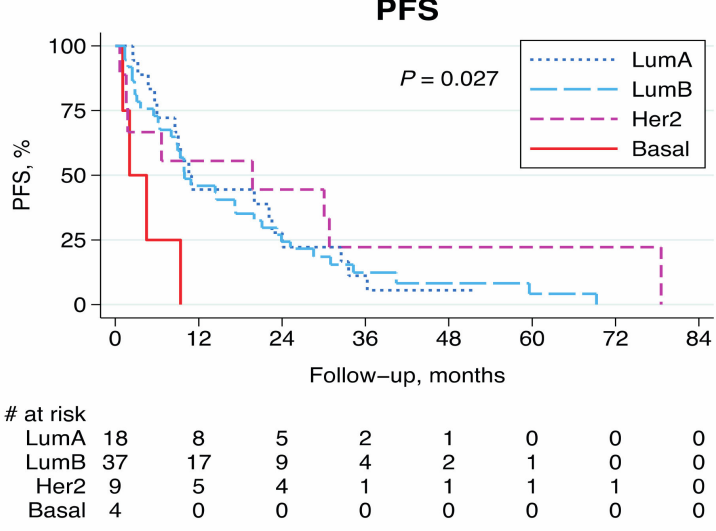

D

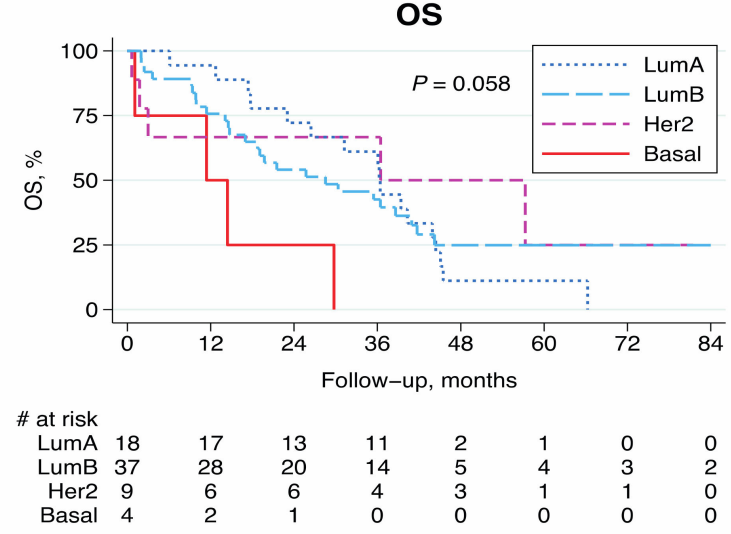

\section{Distant Metastasis}

E

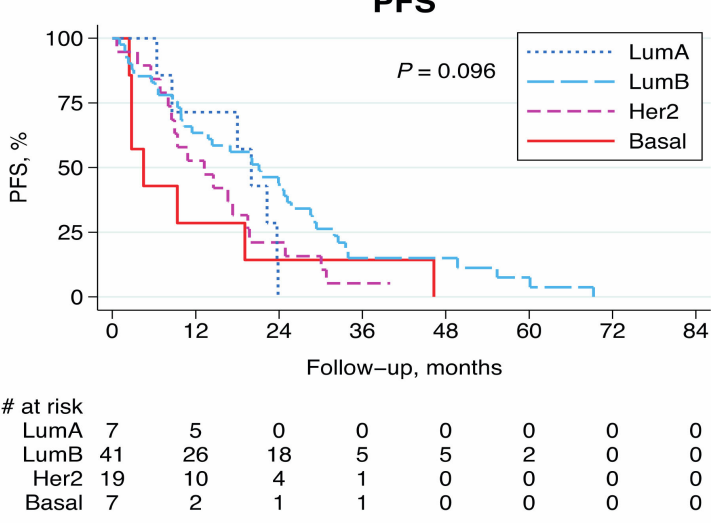

$F$

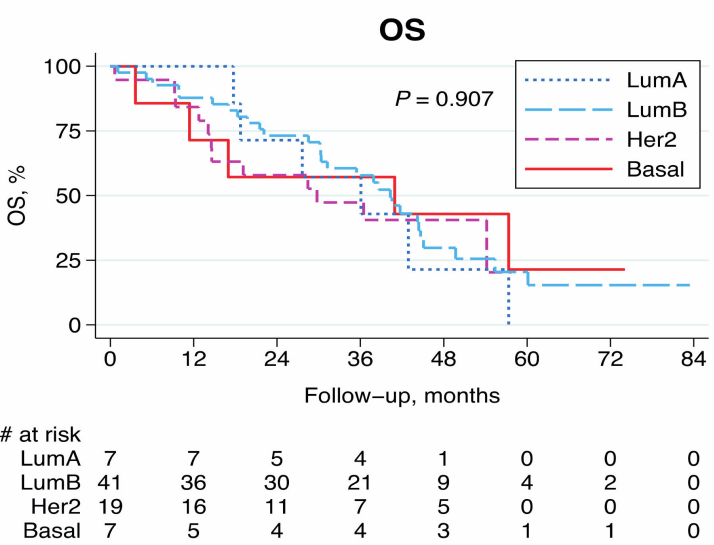

Figure 3. Kaplan-Meier survival curves for progression-free survival (PFS) and overall survival (OS) according to PAM50 breast cancer intrinsic subtype in the primary tumor $(\mathbf{A}, \mathbf{B})$, lymph node metastasis $(\mathbf{C}, \mathbf{D})$, and distant metastasis $(\mathbf{E}, \mathbf{F})$. $p$-values were calculated using the log-rank test. Abbreviations: HER2: human epidermal growth factor receptor 2; Lum A/B: luminal A/B. 
Table 2. Univariable and multivariable Cox regression models of PAM50 breast cancer intrinsic subtypes in primary tumors, lymph node metastases, and distant metastases.

\begin{tabular}{|c|c|c|c|c|c|c|c|c|c|c|c|c|}
\hline & \multicolumn{3}{|c|}{ Univariable PFS } & \multicolumn{3}{|c|}{ Multivariable PFS a } & \multicolumn{3}{|c|}{ Univariable OS } & \multicolumn{3}{|c|}{ Multivariable OS ${ }^{a}$} \\
\hline & $n$ & $\begin{array}{c}\text { HR } \\
(95 \% \text { CI })\end{array}$ & $p$ & $n$ & $\begin{array}{c}\text { HR } \\
(95 \% \text { CI })\end{array}$ & $p$ & $n$ & $\begin{array}{c}\text { HR } \\
(95 \% \mathrm{CI})\end{array}$ & $p$ & $n$ & $\begin{array}{c}\text { HR } \\
(95 \% \mathrm{CI})\end{array}$ & $p$ \\
\hline PT & 123 & & & 89 & & & 123 & & & 69 & & \\
\hline $\begin{array}{l}\text { PAM50 } \\
\text { subtype }\end{array}$ & & & $0.002^{b}$ & & & $0.007^{b}$ & & & $0.001^{b}$ & & & $0.001^{b}$ \\
\hline Lum A & 47 & 1.00 & & & 1.00 & & & 1.00 & & & 1.00 & \\
\hline Lum B & 45 & $\begin{array}{c}0.94 \\
(0.61-1.4)\end{array}$ & 0.79 & & $\begin{array}{c}0.93 \\
(0.54-1.6)\end{array}$ & 0.81 & & $\begin{array}{c}1.1 \\
(0.66-1.8)\end{array}$ & 0.77 & & $\begin{array}{c}0.92 \\
(0.48-1.8)\end{array}$ & 0.81 \\
\hline HER2-E & 15 & $\begin{array}{c}1.1 \\
(0.58-2.0)\end{array}$ & 0.82 & & $\begin{array}{c}0.88 \\
(0.38-2.0)\end{array}$ & 0.77 & & $\begin{array}{c}1.0 \\
(0.52-2.1)\end{array}$ & 0.92 & & $\begin{array}{c}0.77 \\
(0.31-1.9)\end{array}$ & 0.57 \\
\hline Basal-like & 16 & $\begin{array}{c}2.8 \\
(1.6-5.1)\end{array}$ & 0.001 & & $\begin{array}{c}3.8 \\
(1.5-9.3)\end{array}$ & 0.004 & & $\begin{array}{c}3.4 \\
(1.8-6.3)\end{array}$ & $<0.001$ & & $\begin{array}{c}4.6 \\
(1.8-11.3)\end{array}$ & 0.001 \\
\hline LNM & 68 & & & 55 & & & 68 & & & 44 & & \\
\hline $\begin{array}{l}\text { PAM50 } \\
\text { subtype }\end{array}$ & & & $0.05^{b}$ & & & $0.28^{b}$ & & & $0.09^{b}$ & & & $0.17^{b}$ \\
\hline Lum A & 18 & 1.00 & & & 1.00 & & & 1.00 & & & 1.00 & \\
\hline Lum B & 37 & $\begin{array}{c}1.1 \\
(0.6-1.9)\end{array}$ & 0.84 & & $\begin{array}{c}0.88 \\
(0.41-1.9)\end{array}$ & 0.76 & & $\begin{array}{c}0.93 \\
(0.5-1.7)\end{array}$ & 0.81 & & $\begin{array}{c}1.2 \\
(0.53-2.8)\end{array}$ & 0.63 \\
\hline HER2-E & 9 & $\begin{array}{c}0.73 \\
(0.3-1.8)\end{array}$ & 0.48 & & $\begin{array}{c}0.41 \\
(0.13-1.3)\end{array}$ & 0.12 & & $\begin{array}{c}0.63 \\
(0.23-1.7)\end{array}$ & 0.37 & & $\begin{array}{c}0.45 \\
(0.13-1.6)\end{array}$ & 0.23 \\
\hline Basal-like & 4 & $4.2(1.4-13)$ & 0.01 & & $\begin{array}{c}1.5 \\
(0.37-6.4)\end{array}$ & 0.55 & & $\begin{array}{c}3.4 \\
(1.1-10.3)\end{array}$ & 0.04 & & $\begin{array}{c}2.7 \\
(0.64-11.6)\end{array}$ & 0.18 \\
\hline DM & 74 & & & 58 & & & 74 & & & 42 & & \\
\hline $\begin{array}{l}\text { PAM50 } \\
\text { subtype }\end{array}$ & & & $0.11^{b}$ & & & $0.002^{b}$ & & & $0.91^{\mathrm{b}}$ & & & $0.05^{\mathrm{b}}$ \\
\hline Lum A & 7 & 1.00 & & & 1.00 & & & 1.00 & & & 1.00 & \\
\hline Lum B & 41 & $\begin{array}{c}0.60 \\
(0.26-1.4)\end{array}$ & 0.22 & & $\begin{array}{c}0.26 \\
(0.08-0.80)\end{array}$ & 0.02 & & $\begin{array}{c}0.77 \\
(0.32-1.9)\end{array}$ & 0.56 & & $\begin{array}{c}0.46 \\
(0.13-1.6)\end{array}$ & 0.22 \\
\hline HER2-E & 19 & $\begin{array}{c}1.0 \\
(0.42-2.5)\end{array}$ & 0.97 & & $\begin{array}{c}0.64 \\
(0.20-2.1)\end{array}$ & 0.46 & & $\begin{array}{c}0.88 \\
(0.33-2.4)\end{array}$ & 0.81 & & $\begin{array}{c}1.6 \\
(0.40-6.8)\end{array}$ & 0.49 \\
\hline Basal-like & 7 & $\begin{array}{c}1.4 \\
(0.46-3.9)\end{array}$ & 0.58 & & $\begin{array}{c}2.4 \\
(0.54-10.3)\end{array}$ & 0.26 & & $\begin{array}{c}0.69 \\
(0.21-2.3)\end{array}$ & 0.55 & & $\begin{array}{c}1.3 \\
(0.24-6.4)\end{array}$ & 0.79 \\
\hline
\end{tabular}

Abbreviations: CI: confidence interval; DM: distant metastasis; ECOG: Eastern Cooperative Oncology Group; HR: hazard ratio; HER2-E: human epidermal growth factor receptor 2 enriched; LNM: lymph node metastasis; MFI: metastasis-free interval; NHG: Nottingham Histological Grade; $n$ : number; OS: overall survival; PT: primary tumor; PFS: progression-free survival. ${ }^{\text {a }}$ Adjusted for age at diagnosis of metastatic breast cancer ( $<65$ versus $\geq 65$ years), ECOG performance status ( 0 versus 1 versus 2$)$, NHG of PT (I versus II versus III), MFI ( 0 versus $>0-3$ versus $>3$ years), number of metastatic sites ( $<3$ versus $\geq 3$ ), site of metastasis (visceral versus non-visceral), and circulating tumor cell (CTC) status ( $<5$ versus $\geq 5 \mathrm{CTCs}) ;$ b 3 -degree-of-freedom overall test.

\subsection{PAM50 Breast Cancer Intrinsic Subtype Shifts and Outcome in Patients with Paired Samples}

In comparing tumors with shifts between luminal and non-luminal subtypes (luminal to non-luminal and non-luminal to luminal) versus tumors with a stable subtype (luminal stable and non-luminal stable), suggestive evidence of an association between these subgroups and PFS was seen between matched PT and LNM samples $(n=59)(p=0.01$, log-rank test; Figure S1A) and between matched PT and DM samples $(n=61)(p=0.03$, log-rank test; Figure S1C).

Patients with a shift from a luminal PT subtype to a non-luminal DM subtype $(n=12)$ had an inferior PFS compared with patients with a stable luminal subtype $(n=40$, log-rank; HR: 2.31; 95\% CI: 1.14-4.68, $p=0.02$; Figure 4A). The same tendency was seen regarding the OS when comparing patients with a stable luminal subtype to patients with a shift from a luminal-like subtype in the PT to a non-luminal subtype in the DM (log-rank; HR: 1.86; CI: $0.87-4.0, p=0.11$; Figure $4 B$ ). 


\section{Primary Tumor versus Distant Metastasis}

A

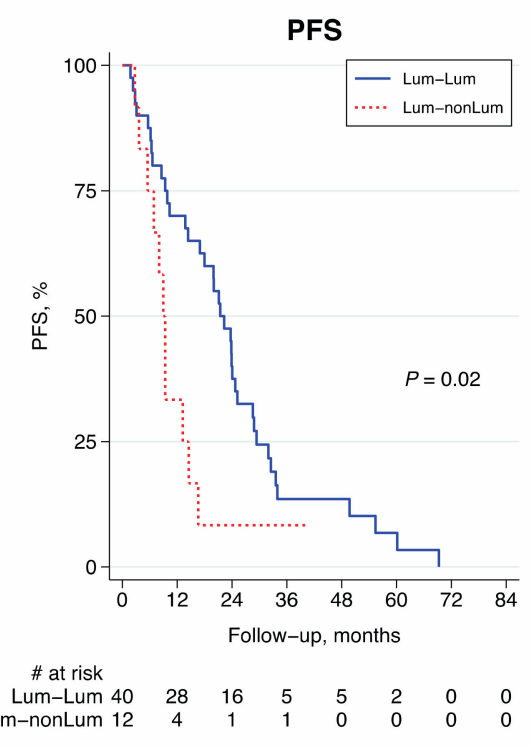

B

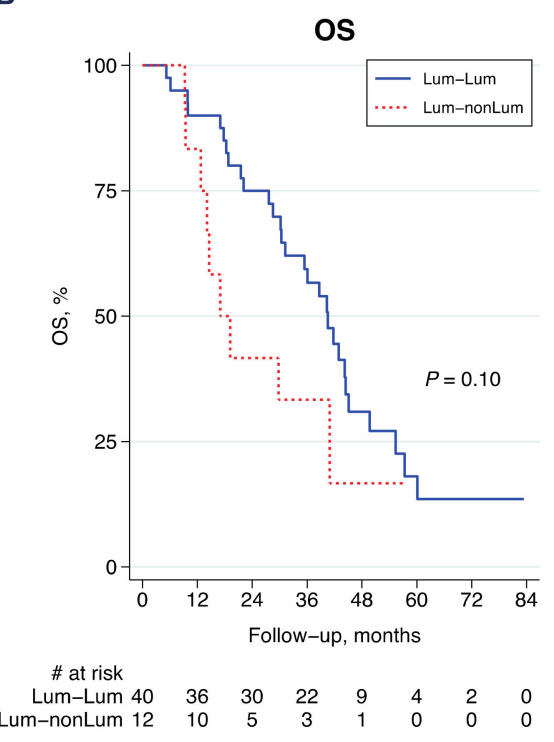

Figure 4. Kaplan-Meier survival curves for (A) progression-free survival (PFS) and (B) overall survival (OS) in relation to subgroups of patients with shifts between luminal (luminal A or B (Lum)) and non-luminal (HER2-E or basal-like (nonLum)) subtypes from the primary tumor to distant metastasis. $p$-values were calculated using the log-rank test.

\section{Discussion}

To test the hypothesis that molecular subtypes are inconsistent during tumor progression in MBC patients, we investigated PAM50 intrinsic breast cancer subtypes in samples of PTs, synchronous LNMs, and DMs. We found strong evidence that PAM50 breast cancer intrinsic subtypes are unstable during tumor progression. Breast cancer intrinsic subtype shifts of $42-51 \%$ were detected between tumor progression sites, and the majority of subtype shifts were toward more aggressive subtypes in metastases. The observed shift towards an unfavorable subtype from PT to DM confirms previous data regarding biomarker status, whereas the significant shift toward a more aggressive molecular subtype in synchronous LNMs has, to our knowledge, not been previously described [9-11]. The pronounced conversion from a luminal PT subtype to a non-luminal DM subtype was associated with a worse prognosis, underscoring the relevance of the re-evaluation of the molecular subtypes in metastatic biopsies.

Previous studies have compared breast cancer molecular subtypes between tumor progression sites by means of IHC-based St Gallen classification [9-11] or using PAM50 gene expression signatures [9,12-16]. Similar to our results, these publications found that while a large portion of paired (matched) samples maintained the same molecular subtype, shifts also occurred over time in multiple cases, with a trend of primarily changing from the luminal A subtype to more unfavorable subtypes.

The reason why these shifts in molecular subtype inherence occur between tumor sites remains speculative. However, in line with our and previous results, a recently published mathematical cancer progression model developed by Chen and colleagues that included data from matched primary and metastatic tumor samples supports a directional linear evolution through luminal subtypes to increasingly aggressive subtypes [15]. We also detected subtype shifts in the other direction, i.e., the luminal B and HER2-E subtypes shifting to the luminal A subtype, emphasizing the possible plasticity and complexity of tumor progression and cancer evolution. Other explanations could be intratumor 
heterogeneity and the fact that multiple breast cancer subtypes co-exist within the same tumor [26,27].

This is supported the work of by Lluch et al. [16], who found an association between clinical subtype changes and dynamic clonal remodeling in breast cancer metastases. In addition, a shift in molecular status may mirror changes following adjuvant therapy [28]. We did see an association between PAM50 subtype shifts and adjuvant endocrine treatment, indicating the possibility that tumor cells that survive adjuvant therapy are likely to be endocrine-resistant clones that are more able to metastasize (e.g., non-luminal subtypes). The determined association reflects the fact that cases where there is a shift in subtype between tumor sites are most often classified as luminal A PT subtypes. Additionally, the significant shift in the PAM50 subtype between PTs and synchronous LNMs suggests that subtype shifts could be independent of treatment effects. It is also plausible that several of the abovementioned factors could occur concurrently.

It is intriguing that the luminal A subtype appeared to be the most unstable subtype throughout tumor progression given that this is the most frequently observed subtype in early breast cancer and is associated with superior prognosis. In addition, patients with this subtype are less heavily treated compared to patients with other subtypes [29-31]. However, the cohort in our study represented patients with metastatic disease, where most cases had node positive disease at the initial diagnosis: hence, our study is not representative of all early breast cancer cases with the luminal A subtype.

In this context, it is important to note that the luminal A subtype has been associated with the highest diversity in clinical outcomes as well as with the highest inter- and even intratumor heterogeneity $[26,32,33]$. A recent study reported worse outcomes in patients with a mixed subclonal luminal A subtype compared to patients with a more 'pure' luminal A subtype [26]. Taken together with our results, these findings highlight the need to stratify the luminal A subtype even further to be able to optimize treatment for these patients.

The shifts in tumor inherence observed in the present study suggested that the intrinsic molecular subtyping of metastatic disease provides additional predictive information that can be used to determine prognosis and therapy. The shift from luminal to non-luminal subtypes observed between PTs and synchronous LNMs (Figure 2A) indicated that this change might be an important consideration when selecting adjuvant treatment. Importantly, patients in which there is conversion from a luminal subtype in the PT to the HER2-E subtype in DMs may benefit from anti-HER2 treatment, as was previously suggested for clinically HER2-negative MBC [34], again highlighting the potential importance of the molecular characterization of metastatic disease. In the present study, we found a suggestive significant difference in PFS between the subgroups of patients with a stable luminal subtype and those with a shift from a luminal subtype in the PT to a non-luminal subtype in DM.

Many studies have focused on the importance of PAM50 subtyping in the early stages of breast cancer, whereas few studies have investigated the prognostic value of PAM50 subtyping in the metastatic setting and instead mostly evaluated prognosis in relation to the PAM50 subtype of the primary tumor $[9,12,34-36]$. The present study has demonstrated the added prognostic value of determining the PAM50 breast cancer intrinsic PT subtype in patients with MBC $[9,13,35,36]$. In line with our findings, Cejalvo et al. found that PAM50 intrinsic PT subtyping had a much greater impact on the overall survival than the subtyping of DMs [13].

The presence of luminal B and HER2-E subtypes during tumor progression in the present study corresponds to the findings of Cejalvo et al. and might represent tumors that lose their estrogen dependence while acquiring a worse phenotype [13]. Interestingly, a recently proposed prognostic model for ER-positive/HER2-negative MBC by Prat et al. combined clinical variables and PAM50 subtyping that was adjusted for the type of biopsy (primary versus metastasis), and it showed a high discriminatory property for predicting prognosis. Their data also supported that PAM50 subtyping adds prognostic information 
in MBC [37]. The prognostic value of subtyping LNMs according to the St Gallen molecular subtype has previously been reported $[9,11]$.

However, we were not able to demonstrate a similar strong prognostic value for subtyping LNMs using the PAM50 subtype classifier. Tobin et al. [38,39] evaluated PAM50 using fine-needle aspirates from synchronous LNMs and asynchronous DMs in relation to post-relapse survival and found that the PAM50 signature was an independent prognostic factor when considering LNMs. One important difference between the present study and the study presented by Tobin et al. is that our study included previously systemically untreated patients with distant metastasis and not those with locoregional disease.

The present study had several strengths. Primarily, it was one of the largest studies to evaluate the PAM50 intrinsic subtype classification of samples, including distant metastases, from a cohort of newly diagnosed $\mathrm{MBC}$ patients who were previously untreated for metastatic disease and who were included in a prospective observational study. All multivariable models of PFS and OS were adjusted for relevant prognostic factors used in clinical practice, as suggested by Bidard et al. [22]. We included samples from $90 \%$ of the participants in the original trial, applied the validated PAM50 intrinsic subtyping assay in a highly standardized manner, and prospectively defined hypotheses and a statistical analysis plan.

However, the study also had certain limitations. The study had a prospectiveretrospective design, where patients had received various systemic treatments, and some of the subgroup analyses had limited statistical power due to the small group size. Even though all included patients had metastatic disease, distant metastatic biopsies were not always available as FFPE samples because the diagnosis of some patients had only been confirmed using computed tomography or cytology examinations. In addition, several patients had more than one synchronous LNM and/or DM; however, we only evaluated one LNM and the first DM in the present analysis. This could have contributed to sampling error and selection bias because the evaluated metastasis might not represent the full metastatic burden in the patient. Finally, studying the dynamic process of tumor progression in momentary 'snapshots' of tumor biopsies at different stages only reflects part of the picture.

\section{Conclusions}

The results of this study confirmed PAM50 molecular subtype shifts during tumor progression. The directional tumor evolution from luminal subtypes to more unfavorable subtypes was shown, indicating the need for the proper molecular characterization of metastatic disease to improve the personalization of systemic therapy. Importantly, the prognosis in patients displaying shifts from luminal subtypes in PTs to basal-like and HER2-enriched inherence in DMs was inferior to that of patients with a stable luminal subtype. A shift in the breast cancer molecular subtype during tumor progression adds predictive information that is clinically relevant for the determination of prognosis and treatment.

Supplementary Materials: The following are available online at https: / www.mdpi.com/article/10 .3390/cancers13071592/s1, Figure S1: Kaplan-Meier survival curves for progression-free survival and overall survival according to luminal versus non-luminal subtype subgroups in the primary tumor, lymph node metastasis, and distant metastasis. $p$-values were calculated with the log-rank test, Table S1: Patient demographics, disease characteristics, and prior therapy given for the entire CTC-MBC cohort and for patients included/excluded in the present study, Table S2: Patient and tumor characteristics for the lymph node metastases in the PAM50 cohort with subtype available and subdivided into the different breast cancer intrinsic subtypes, Table S3: Patient and tumor characteristics for the distant metastases in the PAM50 cohort with subtype available and subdivided into the different breast cancer intrinsic subtypes, Table S4: Changes in PAM50 breast cancer intrinsic subtype between PT, LNM, and DM, Table S5: Overall PAM50 breast cancer intrinsic subtype shifts from primary tumors to distant metastases in relation to adjuvant therapy given. 
Author Contributions: Conceptualization, C.L.T.J., A.-M.L., and L.R.; methodology, C.L.T.J., A.-M.L., C.F., K.A., and L.R.; formal analysis, C.L.T.J., P.-O.B., and R.B.; investigation, C.L.T.J., C.F., A.-M.L., K.A., and S.J.; resources, L.R.; data curation, C.L.T.J., P.-O.B., A.-M.L., and R.B.; writing-originaldraft preparation, C.L.T.J., A.-M.L., C.F., and L.R.; writing-review and editing, all authors; visualization, C.L.T.J., P.-O.B., and C.F.; project administration, C.L.T.J., and L.R.; funding acquisition, L.R. All authors have read and agreed to the published version of the manuscript.

Funding: This work was supported by the BioCare-strategic research area at Lund University; the Swedish Cancer Society $(2016 / 563 ; 190388)$; the Mrs. Berta Kamprad Foundation (2017-39); The Crafoord Foundation (20170702); Governmental Funding of Clinical Research within National Health Service (ALF) (2018-40304; 2020-40634); the Swedish Research Council 2015-02516; and Skåne University Hospital Funds (2017-92502; 2018-92502).

Institutional Review Board Statement: The Lund University Ethics Committee (LU 2010/135) approved the study prior to its initiation.

Informed Consent Statement: Informed consent was obtained from all patients included in the study.

Data Availability Statement: The datasets used and/or analyzed during the current study are available from the corresponding author upon reasonable request.

Acknowledgments: The authors are indebted to Annette Ahlin Gullers, Jessica Åkesson, Emma Edvik, Lina Zander, Petra Andersson, Kristina Lövgren, and the clinical oncologists at Skåne University Hospital and Halmstad County Hospital for their work with the inclusion of patients during the study period of the main study of which the present study was based on. The authors thank Sara Baker for professional construction of the study database and Ulrik Narbe for providing follow-up data. The authors thank Biobank Syd for excellent service with the retrieval of FFPE tissue, clinical pathologist Anna Ehinger for pathology consultancy, and Ralph Schulz for guidance and participation in the initial steps of the study.

Conflicts of Interest: R.B. is employed by NanoString Technologies. All other authors declare no conflict of interest.

\section{References}

1. Dillekås, H.; Rogers, M.S.; Straume, O. Are 90\% of deaths from cancer caused by metastases? Cancer Med. 2019, 8, 5574-5576. [CrossRef] [PubMed]

2. Davis, A.; Gao, R.; Navin, N. Tumor evolution: Linear, branching, neutral or punctuated? Biochim. Biophys. Acta Bioenerg. 2017, 1867, 151-161. [CrossRef] [PubMed]

3. Joseph, C.; Papadaki, A.; Althobiti, M.; Alsaleem, M.; Aleskandarany, M.A.; Rakha, E.A. Breast cancer intratumour heterogeneity: Current status and clinical implications. Histopathology 2018, 73, 717-731. [CrossRef]

4. Kimbung, S.; Loman, N.; Hedenfalk, I. Clinical and molecular complexity of breast cancer metastases. Semin. Cancer Biol. 2015, 35, 85-95. [CrossRef]

5. Aurilio, G.; Disalvatore, D.; Pruneri, G.; Bagnardi, V.; Viale, G.; Curigliano, G.; Adamoli, L.; Munzone, E.; Sciandivasci, A.; De Vita, F.; et al. A meta-analysis of oestrogen receptor, progesterone receptor and human epidermal growth factor receptor 2 discordance between primary breast cancer and metastases. Eur. J. Cancer 2014, 50, 277-289. [CrossRef] [PubMed]

6. Cummings, M.C.; Simpson, P.T.; Reid, L.E.; Jayanthan, J.; Skerman, J.; Song, S.; Reed, A.E.M.; Kutasovic, J.R.; Morey, A.L.; Marquart, L.; et al. Metastatic progression of breast cancer: Insights from 50 years of autopsies. J. Pathol. 2014, $232,23-31$. [CrossRef]

7. Schrijver, W.; Suijkerbuijk, K.P.M.; van Gils, C.H.; van der Wall, E.; Moelans, C.B.; van Diest, P.J. Receptor Conversion in Distant Breast Cancer Metastases: A Systematic Review and Meta-analysis. J. Natl. Cancer Inst. 2018, 110, 568-580. [CrossRef]

8. Cardoso, F.; Senkus, E.; Costa, A.; Papadopoulos, E.; Aapro, M.; Andre, F.; Harbeck, N.; Lopez, B.A.; Barrios, C.H.; Bergh, J.; et al. 4th ESO-ESMO International Consensus Guidelines for Advanced Breast Cancer (ABC 4) dagger. Ann. Oncol. 2018, 29, $1634-1657$. [CrossRef] [PubMed]

9. Kimbung, S.; Kovács, A.; Danielsson, A.; Bendahl, P.-O.; Lövgren, K.; Stolt, M.F.; Tobin, N.P.; Lindström, L.; Bergh, J.; Einbeigi, Z.; et al. Contrasting breast cancer molecular subtypes across serial tumor progression stages: Biological and prognostic implications. Oncotarget 2015, 6, 33306-33318. [CrossRef] [PubMed]

10. Falck, A.K.; Bendahl, P.O.; Chebil, G.; Olsson, H.; Ferno, M.; Ryden, L. Biomarker expression and St Gallen molecular subtype classification in primary tumours, synchronous lymph node metastases and asynchronous relapses in primary breast cancer patients with 10 years' follow-up. Breast Cancer Res. Treat. 2013, 140, 93-104. [CrossRef] [PubMed]

11. Falck, A.K.; Ferno, M.; Bendahl, P.O.; Ryden, L. St Gallen molecular subtypes in primary breast cancer and matched lymph node metastases-Aspects on distribution and prognosis for patients with luminal A tumours: Results from a prospective randomised trial. BMC Cancer 2013, 13, 558. [CrossRef] 
12. Lee, J.Y.; Park, K.; Lee, E.; Ahn, T.; Jung, H.H.; Lim, S.H.; Hong, M.; Do, I.-G.; Cho, E.Y.; Kim, D.-H.; et al. Gene Expression Profiling of Breast Cancer Brain Metastasis. Sci. Rep. 2016, 6, 28623. [CrossRef]

13. Cejalvo, J.M.; De Dueñas, E.M.; Galván, P.; García-Recio, S.; Gasión, O.B.; Paré, L.; Antolín, S.; Martinello, R.; Blancas, I.; Adamo, B.; et al. Intrinsic Subtypes and Gene Expression Profiles in Primary and Metastatic Breast Cancer. Cancer Res. 2017, 77, $2213-2221$. [CrossRef] [PubMed]

14. Priedigkeit, N.; Hartmaier, R.J.; Chen, Y.; Vareslija, D.; Basudan, A.; Watters, R.J.; Thomas, R.; Leone, J.P.; Lucas, P.C.; Bhargava, R.; et al. Intrinsic Subtype Switching and Acquired ERBB2/HER2 Amplifications and Mutations in Breast Cancer Brain Metastases. JAMA Oncol. 2017, 3, 666-671. [CrossRef]

15. Chen, R.; Goodison, S.; Sun, Y. Molecular profiles of matched primary and metastatic tumor samples support a linear evolutionary model of breast cancer. Cancer Res. 2020, 80. [CrossRef]

16. Lluch, A.; Gonzalez-Angulo, A.M.; Casadevall, D.; Eterovic, A.K.; de Duenas, E.M.; Zheng, X.; Guerrero-Zotano, Á.; Liu, S.; Pérez, R.; Chen, K.; et al. Dynamic clonal remodelling in breast cancer metastases is associated with subtype conversion. Eur. J. Cancer 2019, 120, 54-64. [CrossRef]

17. Woo, J.W.; Chung, Y.R.; Ahn, S.; Kang, E.; Kim, E.-K.; Kim, S.H.; Kim, J.H.; Kim, I.A.; Park, S.Y. Changes in Biomarker Status in Metastatic Breast Cancer and Their Prognostic Value. J. Breast Cancer 2019, 22, 439-452. [CrossRef] [PubMed]

18. Simon, R.M.; Paik, S.; Hayes, D.F. Use of Archived Specimens in Evaluation of Prognostic and Predictive Biomarkers. J. Natl. Cancer Inst. 2009, 101, 1446-1452. [CrossRef]

19. Larsson, A.-M.; Jansson, S.; Bendahl, P.-O.; Jörgensen, C.L.T.; Loman, N.; Graffman, C.; Lundgren, L.; Aaltonen, K.; Rydén, L. Longitudinal enumeration and cluster evaluation of circulating tumor cells improve prognostication for patients with newly diagnosed metastatic breast cancer in a prospective observational trial. Breast Cancer Res. 2018, 20, 48. [CrossRef] [PubMed]

20. Geiss, G.K.; Bumgarner, R.E.; Birditt, B.; Dahl, T.; Dowidar, N.; Dunaway, D.L.; Fell, H.P.; Ferree, S.; George, R.D.; Grogan, T.; et al. Direct multiplexed measurement of gene ex-pression with color-coded probe pairs. Nat. Biotechnol. 2008, 26, 317-325. [CrossRef]

21. Parker, J.S.; Mullins, M.; Cheang, M.C.; Leung, S.; Voduc, D.; Vickery, T.; Davies, S.; Fauron, C.; He, X.; Hu, Z.; et al. Supervised Risk Predictor of Breast Cancer Based on Intrinsic Subtypes. J. Clin. Oncol. 2009, 27, 1160-1167. [CrossRef]

22. Bidard, F.-C.; Peeters, D.J.; Fehm, T.; Nolé, F.; Gisbert-Criado, R.; Mavroudis, D.; Grisanti, S.; Generali, D.; Garcia-Saenz, J.A.; Stebbing, J.; et al. Clinical validity of circulating tumour cells in patients with metastatic breast cancer: A pooled analysis of individual patient data. Lancet Oncol. 2014, 15, 406-414. [CrossRef]

23. Benjamin, D.J.; Berger, J.O.; Johannesson, M.; Nosek, B.A.; Wagenmakers, E.-J.; Berk, R.; Bollen, K.A.; Brembs, B.; Brown, L.; Camerer, C.; et al. Redefine statistical significance. Nat. Hum. Behav. 2018, 2, 6-10. [CrossRef] [PubMed]

24. Altman, D.G.; McShane, L.M.; Sauerbrei, W.; Taube, S.E. Reporting Recommendations for Tumor Marker Prognostic Studies (REMARK): Explanation and Elaboration. PLoS Med. 2012, 9, e1001216. [CrossRef]

25. McShane, L.M.; Altman, D.G.; Sauerbrei, W.; Taube, S.E.; Gion, M.; Clark, G.M. Reporting recommendations for tumor MARK-er prognostic studies (REMARK). Breast Cancer Res. Treat. 2006, 100, 229-235. [CrossRef]

26. Kumar, N.; Zhao, D.; Bhaumik, D.; Sethi, A.; Gann, P.H. Quantification of intrinsic subtype ambiguity in Luminal A breast cancer and its relationship to clinical outcomes. BMC Cancer 2019, 19, 215. [CrossRef]

27. Yeo, S.K.; Guan, J.-L. Breast Cancer: Multiple Subtypes within a Tumor? Trends Cancer 2017, 3, 753-760. [CrossRef]

28. Turner, N.C.; Reis-Filho, J.S. Genetic heterogeneity and cancer drug resistance. Lancet Oncol. 2012, 13, e178-e185. [CrossRef]

29. Sorlie, T.; Perou, C.M.; Tibshirani, R.; Aas, T.; Geisler, S.; Johnsen, H.; Hastie, T.; Eisen, M.B.; van de Rijn, M.; Jeffrey, S.S.; et al. Gene expression patterns of breast carcinomas distinguish tumor subclasses with clinical implications. Proc. Natl. Acad. Sci. USA 2001, 98, 10869-10874. [CrossRef]

30. Perou, C.M.; Sørlie, T.; Eisen, M.B.; Van De Rijn, M.; Jeffrey, S.S.; Rees, C.A.; Pollack, J.R.; Ross, D.T.; Johnsen, H.; Akslen, L.A.; et al. Molecular portraits of human breast tumours. Nature 2000, 406, 747-752. [CrossRef]

31. Sorlie, T.; Tibshirani, R.; Parker, J.; Hastie, T.; Marron, J.S.; Nobel, A.; Deng, S.; Johnsen, H.; Pesich, R.; Geisler, S.; et al. Repeated observation of breast tumor subtypes in independent gene expression data sets. Proc. Natl. Acad. Sci. USA 2003, 100, 8418-8423. [CrossRef]

32. Jiang, T.; Shi, W.; Natowicz, R.; Ononye, S.N.; Wali, V.B.; Kluger, Y.; Pusztai, L.; Hatzis, C. Statistical measures of transcriptional diversity capture genomic heterogeneity of cancer. BMC Genom. 2014, 15, 876. [CrossRef] [PubMed]

33. Ciriello, G.; Sinha, R.; Hoadley, K.A.; Jacobsen, A.S.; Reva, B.; Perou, C.M.; Sander, C.; Schultz, N. The molecular diversity of Luminal A breast tumors. Breast Cancer Res. Treat. 2013, 141, 409-420. [CrossRef]

34. Prat, A.; Cheang, M.C.; Galvan, P.; Nuciforo, P.; Pare, L.; Adamo, B.; Muñoz, M.; Viladot, M.; Press, M.F.; Gagnon, R.; et al. Prognostic Value of Intrinsic Subtypes in Hormone Receptor-Positive Metastatic Breast Cancer Treated with Letrozole with or without Lapatinib. JAMA Oncol. 2016, 2, 1287-1294. [CrossRef]

35. Jørgensen, C.; Nielsen, T.; Bjerre, K.; Liu, S.; Wallden, B.; Balslev, E.; Nielsen, D.; Ejlertsen, B. Abstract P3-06-03: Association between PAM50 breast cancer intrinsic subtypes and effect of gemcitabine in advanced breast cancer patients. Poster Sess. Abstr. 2012, 53, 776-787. [CrossRef]

36. Falato, C.; Tobin, N.P.; Lorent, J.; Lindström, L.S.; Bergh, J.; Foukakis, T. Intrinsic subtypes and genomic signatures of primary breast cancer and prognosis after systemic relapse. Mol. Oncol. 2015, 10, 517-525. [CrossRef] [PubMed] 
37. Prat, A.; Tsai, Y.-H.; Pascual, T.; Paré, L.; Adamo, B.; Vidal, M.; Brasó-Maristany, F.; Galván, P.; Brase, J.C.; Rodrik-Outmezguine, V.; et al. A Prognostic Model Based on PAM50 and Clinical Variables (PAM50MET) for Metastatic Hormone Receptor-positive HER2-negative Breast Cancer. Clin. Cancer Res. 2020, 26, 6141-6148. [CrossRef]

38. Tobin, N.P.; Harrell, J.C.; Lovrot, J.; Brage, S.E.; Stolt, M.F.; Carlsson, L.; Einbeigi, Z.; Linderholm, B.; Loman, N.; Malmberg, M.; et al. Molecular subtype and tumor characteristics of breast cancer metastases as assessed by gene expression significantly influence patient post-relapse survival. Ann. Oncol. 2015, 26, 81-88. [CrossRef]

39. Tobin, N.P.; Lundberg, A.; Lindström, L.S.; Harrell, J.C.; Foukakis, T.; Carlsson, L.; Einbeigi, Z.; Linderholm, B.K.; Loman, N.; Malmberg, M.; et al. PAM50 Provides Prognostic Information When Applied to the Lymph Node Metastases of Advanced Breast Cancer Patients. Clin. Cancer Res. 2017, 23, 7225-7231. [CrossRef] 